\title{
Diagnostic effectiveness of noncontact slitlamp examination in the identification of retinal tears
}

\begin{abstract}
Purpose To compare the diagnostic accuracy of noncontact slitlamp examination with indirect ophthalmoscopy and scleral depression, in the identification of retinal tears.

Methods A prospective study was performed using 17 patients who were referred with retinal tears. All were initially examined at the slitlamp with a hand-held Volk (noncontact) lens. The same observer then carried out indirect ophthalmoscopy with scleral indentation.

Results In 17 eyes a total of 18 acute retinal u-tears were found. A total of $\mathbf{1 6}$ tears were picked up at the initial examination at the slitlamp (89\%), while two were missed $(11 \%)$. Conclusions Indirect ophthalmoscopy with scleral depression is the 'gold standard' for the identification of peripheral retinal tears. This small study has shown that although the majority of these can be picked up by the use of a noncontact lens at the slitlamp, $11 \%$ were missed using this technique.

Eye (2003) 17, 607-609. doi:10.1038/

sj.eye. 6700456
\end{abstract}

Keywords: retinal tear; slitlamp; indirect ophthalmoscope

\section{Introduction}

A retinal tear is a well-recognised complication following separation of the cortical vitreous and posterior hyaloid face from the inner retina. ${ }^{1}$ This detachment of the posterior vitreous may manifest itself as a sudden onset of flashes and/ or floaters, thus prompting patients to be referred to ophthalmic departments.
M Natkunarajah, C Goldsmith and R Goble

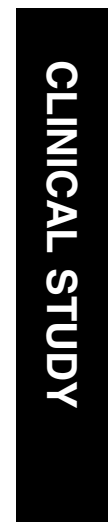

Retinal tears have been found to occur in $10-15 \%$ of patients who present with an acute symptomatic posterior vitreous detachment. ${ }^{2}$ Their identification is important, as they have been found to lead to the development of a retinal detachment in $33-46 \%$ of cases. ${ }^{3}$ The 'gold standard' for the diagnosis of retinal tears is the use of the indirect ophthalmoscope combined with scleral depression. In cases where the view is adequate, indirect ophthalmoscopy has been found to identify all peripheral tears at the time of presentation, so obviating the need for prolonged follow-up. ${ }^{4}$ However, it is a technique that takes time to master by ophthalmologists in training. This small study was carried out to look at the accuracy of using slitlamp examination with the use of a hand-held Volk lens in the identification of peripheral retinal tears compared with the use of indirect ophthalmoscopy with scleral depression.

\section{Methods}

Patients presenting with symptoms of flashes and floaters of less than a month's duration were examined by a junior member of staff during a 12-month period between August 2000 and September 2001. Those who were found to have a retinal tear were prospectively recruited.

A single observer (a consultant vitreoretinal surgeon) who was masked to the previous findings of the number and location of retinal tears subsequently examined all patients. If at any stage the observer became unmasked, the patient was excluded from the study. Patients were dilated with $1 \%$ tropicamide and $2.5 \%$ phenylephrine prior to consultation.

A two-stage evaluation was carried out; firstly slitlamp examination of the peripheral
Ipswich Hospital

Ipswich, Suffolk, UK

Correspondence: R Goble Ipswich Hospital Ipswich, Suffolk IP4 5PD, UK

Tel.: +44 1473703504 Fax: + 441473703528

E-mail: goble.sec@

ipsh_tr.anglox.nhs.uk

Received: 5 June 2002 Accepted in revised form: 1 November 2002

Paper presented at Beavrs, Brighton, October 2001 by Dr R Goble 
retina in the nine positions of gaze using a hand-held Volk Superfield. The second stage involved indirect ophthalmoscopy using a $28 \mathrm{D}$ condensing lens and $360^{\circ}$ scleral depression with the patient supine. The findings of the two examination techniques carried out by the consultant were then compared.

Patients who had evidence of a retinal detachment or atrophic holes were excluded from the study, as were those who did not have a tear identified by either method. A total of 17 patients were found to have a retinal tear and thus recruited into this study.

\section{Results}

We studied 17 eyes of 17 patients. Eight were women female with an average age of 58.9 years (range $36-80$ years) and nine were males with an average age of 57.7 years (range $38-68$ years).

A total of 18 retinal tears were diagnosed using the indirect ophthalmoscope, 16 (89\%) of which were correctly identified using the slitlamp. Two retinal tears were missed by slitlamp examination (11\%).

The features of the tears that were missed on slitlamp examination are as follows:

Case 11: The u-tear was very anterior in location and only visible on indentation at the ora serrata.

Case 16: On slitlamp examination the tear was masked in an area of pigmented lattice degeneration. It was only on indentation that the operculum of the u-tear became visible.

These tears were retrospectively looked for with the three-mirror contact lens, but failed to be identified.

No tears were identified using the slitlamp alone.

\section{Discussion}

Retinal tears are a known sequel of posterior vitreous detachments. Their identification is of importance as they can lead to the development of a retinal detachment. The classical symptomatology of an acute posterior vitreous detachment is that of sudden onset of flashes and/or floaters, although their predictive value in relation to a retinal break is not conclusive. ${ }^{5}$ Others have found that these symptoms in association with a subjective decrease in vision to be more predictive. ${ }^{6}$

Examination of the retina in all patients who present with an acute posterior vitreous detachment should be thorough and include the anterior retina. Early identification of breaks can lead to prophylactic treatment by argon photocoagulation or cryotherapy retinopexy, and so lower the risks of a subsequent rhegmatogenous retinal detachment. ${ }^{3}$ All the patients in this study received such prophylactic treatment. None of the patients progressed to develop a retinal detachment.
The most widely used method of examination is the indirect ophthalmoscope and condensing lens with scleral indentation at the ora. However, the slitlamp has its uses and should not be underestimated regarding its effectiveness in the diagnosis of retinal tears, particularly for those untrained in scleral indentation or still learning the use of the indirect ophthalmoscope.

This study, given the limits of a small patient sample, has shown that a high proportion of retinal tears (89\%) themselves can be identified using a noncontact lens at the slitlamp. One advantage of slitlamp examination is the higher level of magnification. However, its limitations are that the anterior retina is not easily visualised. A method of scleral indentation with the patient at the slitlamp has been described in order to overcome this. ${ }^{7}$ Our high pickup rates using the slitlamp may be due to an evaluation bias, as all the patients entered into the study were thought to have a retinal tear. The level of experience of the observer must also be taken into account; similar pickup rates may not have been achieved with less experienced observers.

It must be noted that not all tears were picked up using the slitlamp examination alone, as 2/18 tears were missed. No additional information was obtained using the Vitreofundus lens or the three-mirror lens compared with the Superfield lens. Their identification by use of the indirect ophthalmoscope may have been because one tear was very anterior in location, and the other was better viewed upon indentation in order to lift up the operculum.

\section{Conclusions}

This small study shows that a high proportion of retinal tears associated with a posterior vitreous detachment can be seen on slitlamp examination using a noncontact method. However, it is must be noted that $11 \%$ of retinal tears were missed. Given that up to one-third of these can progress to a retinal detachment, the indirect ophthalmoscope with scleral indentation remains the diagnostic technique of choice.

\section{References}

1 Linder B. Acute posterior vitreous detachment and its retinal complications. Acta Ophthalmol 1966; 87 (Suppl): 1.

2 Novak MA, Welch RB. Complications of acute symptomatic posterior vitreous detachment. Am J Ophthalmol 1984; 97: 308-314.

3 Davis MD. Natural history of retinal breaks without detachment. Arch Ophthalmol 1974; 92: 183-194.

4 Richardson PSR, Benson MT, Kirby GR. The posterior vitreous detachment clinic: do new retinal breaks develop in the six weeks following an isolated symptomatic posterior vitreous detachment? Eye 1999; 13: 237-240. 
5 Tanner V, Harle D, Tan J, Foote B et al. Br J Ophthalmol 2000; 84: $1264-1268$.

6 Dayan MR, Jayamanne DGR, Andrews RM et al. Flashes and floaters as predictors of vitreoretinal pathology: is follow-up necessary for posterior vitreous detachment? Eye 1996; 10: 456-458.
7 Lincoff H, Kreissig I. Finding the retinal hole in the pseudophakic eye with detachment. Am J Ophthalmol 1994; 117: $442-446$ 